\title{
Assessing the potential role of neutrophil elastase as a prognostic indicator in oral squamous cell carcinoma
}

Research Article

\author{
Pooja Jaiswal ${ }^{1}$, Supriya Kheur*1, Pratiksha Mahajan¹, A. Thirumal Raj², \\ Mamatha Reddy ${ }^{1}$, Roopa Madalli ${ }^{1}$ Nitin Gupta ${ }^{1}$ \\ ${ }^{1}$ Department of Oral Pathology and Microbiology, Dr. D. Y. Patil Dental College \\ and Hospital, Dr. D. Y. Patil Vidyapeeth, Pimpri, Pune, India. \\ 2Department of Oral Pathology and Microbiology, Sri Venkateswara Dental \\ College, Chennai, India.
}

Received 10 September 2018; Accepted 25 March 2019

\begin{abstract}
Past decade has seen the advent of several prognosticators for oral squamous cell carcinoma (OSCC). Although TNM staging is still considered as the prognostic gold standard, it is not possible to obtain all the necessary information for a pathological TNM staging until excisional biopsy. Thus, it is necessary to identify features from the initial biopsy, which could aid in early prognostication.

Aim: To compare neutrophil elastase immuno-expression in oral squamous cell carcinoma patients with and without lymph node metastasis.

Materials and Methods: Archival specimens of 50 oral squamous cell carcinoma consisting of 25 cases with and 25 cases without lymph node metastasis were immuno-stained for neutrophil elastase.

Results: Statistically, a significant difference was noted in the neutrophil elastase staining between the 2 groups. 18 of the 25-oral squamous cell carcinoma cases with lymph node metastasis were positive and only 5 of the 25 -oral squamous cell carcinoma cases without nodal involvement were positive.

Conclusion: Presence of neutrophil elastase in the lesional tissue is strongly associated with a lymph node involvement, and thus could serve as a prognostic factor for oral squamous cell carcinoma.
\end{abstract}

Keywords: Leukocyte elastase •lymphatic metastasis • staining and labelling • carcinoma • squamous cell

\section{Introduction}

The development of Oral Squamous Cell Carcinoma or any malignancy is associated with a complex biological crosstalk between tumour cells, stromal cells, and host inflammatory cells. One of the important mechanisms of the tumour cells for invading into the host stroma is the evasion of immune surveillance. The host defense barrier is generally carried out by the neutrophils, macrophages, and lymphocytes. These cells have also been shown to have dual or

interchanging role in tumour immunity. Extensive work carried out on macrophages, in vitro, and in vivo have to lead to the characterization of macrophages into M1 (Tumour killing/activated) and M2 (Tumour promoting/alternatively activated). Thus, macrophages can show a phenotypic switch, which suppresses the immune response against invading malignant epithelial cells. ${ }^{[1]}$ The other type of immune cells are neutrophils, which form the first line of defense against any invading cell. These cells are the key effectors of the innate immune system with prominent antibacterial and antifungal activity. They generally release proteases, which contains Matrix Metalloproteinases -8, -9, ADAM (a disintegrin and metalloproteinase) 8, -17, ADAMTS (a disintegrin metalloproteinase and thrombospondin motifs) -1 , inhibitors of TIMP-1. . $^{[2]}$

Recent evidence suggests that a tumour-associated neutrophil (TAN) can promote tumour progression and metastasis through different mechanisms, including the activation of cytokines, integrin binding, reactive oxygen species formation, secretion of neutrophil elastase (NE) and proteases that directly affect the tumour microenvironment. In prostate cancer, the granulocytic 


\begin{tabular}{|c|c|c|c|c|c|c|c|c|c|}
\hline \multirow{2}{*}{ Study groups } & \multirow{2}{*}{$\begin{array}{c}\text { Average } \\
\text { Age } \\
\text { (in years) }\end{array}$} & \multicolumn{2}{|c|}{ Gender } & \multicolumn{4}{|c|}{ Site } & \multicolumn{2}{|c|}{ Staging } \\
\hline & & Male & Female & $\begin{array}{c}\text { Gingivo-buccal } \\
\text { sulcus }\end{array}$ & Alveolus & Tongue & $\begin{array}{l}\text { Buccal } \\
\text { mucosa }\end{array}$ & III & IV \\
\hline $\begin{array}{l}\text { OSCC with } \\
\text { lymph node } \\
\text { metastasis }\end{array}$ & 52.92 & 16 & 9 & 8 & 10 & 3 & 4 & 18 & 7 \\
\hline $\begin{array}{c}\text { OSCC } \\
\text { without } \\
\text { lymph node } \\
\text { metastasis }\end{array}$ & 53.88 & 17 & 8 & 9 & 11 & 2 & 3 & 19 & 6 \\
\hline
\end{tabular}

myeloid derived suppressor cells have shown to aid in tumour progression through secretion of NE. Given the tumour associated inflammatory component noted in oral cancer, it is possible for NE to play a similar role in oral cancer progression. The presence of TANs is emerging as a prognostic marker and a possible new tool for cancer treatment. ${ }^{[3]}$ A preliminary study found a direct correlation of CD66 + neutrophils with the tumour size. T3-T4 sized tumours showed increased infiltration of neutrophils as compared to the smaller sized tumour. [4] There is no literature available on the correlation between the lesional neutrophil density with local node involvement.

Thus, this study aims to assess the role of NE in OSCC and correlate with various clinicopathological features, especially locoregional lymph node metastasis. With the abovementioned role of $\mathrm{NE}$, we hypothesize that NE expression will be increased in cases of OSCC with regional lymph node metastasis. To prove this hypothesis, we studied the association of immunohistochemical expression of $\mathrm{NE}$ in the OSCC tissues and correlated it with the lymph node involvement in these patients.

\section{Materials and Methods}

The 50 OSCC specimens of cases who had undergone surgical treatment for oral SCC were selected, and clinical information was retrieved from the archival files. The data collected included age, gender, habit history, clinical and histopathological details including lesion location, staging, and treatment details. The study was approved by the scientific and Institutional Ethics committee. Clinical examination in combination with ultrasonography was used to assess potential lymph node involvement. The lymph nodes resected during the surgical excision were subjected to histopathological examination to confirm the lymph node involvement. The sample consisted of 25 OSCC cases with lymph node metastasis and 25 OSCC cases without nodal involvement. Both the groups (with and without lymph node involvement) were matched for age, gender, site, grading, and staging to avoid confounding effect (table 1). All the samples were either in stage III or IV.

Sections of 5 micrometres $(\mu \mathrm{m})$ width of the tissues' specimens were cut and slides were prepared for both routine Hematoxylin and Eosin, and the immunohistochemical staining procedure. Slide samples stained with Haematoxylin and Eosin were reviewed and the histopathological grading was done according to the criteria established by Border et al. ${ }^{[5]}$

$5 \mu \mathrm{m}$ thick paraffin sections were taken on poly-L-lysine- coated glass slides and stained immunohistochemically using the anti-neutrophil elastase antibody (Ab CAM ab 21595, USA).

The tissue sections were treated with Protease XXV at $1 \mathrm{mg} / \mathrm{ml} \mathrm{PBS}$, (Lab Vision Catalog \# AP- 9004) for 5 minutes at $370 c$. Primary Antibody Incubation Time was 30 minutes at room temperature. Antigen retrieval was done by heat-induced epitope retrieval (HIER) method. The immunohistochemical staining of the section by primary mouse antibody neutrophil elastase was carried out with dilution (1:100). The sites were then visualized using diaminobenzidine (DAB) substrate chromogen (Dako) and counterstained with Mayer's Hematoxylin. For positive control, bone marrow tissue was used provided by the manufacturer. For negative control, sections were treated in a similar manner without the primary antibody. A semi-quantitative analysis was done, based on the reports by Lundqvist et al. ${ }^{\left[{ }^{6]}\right.}$ Four high-power fields (400 $\times$ magnification) were analysed and positivity was graded as 0 (no staining), 1 (1-25\% of positive cells), 2 (25-50\%), and 3 (>50\%) (Figure 1). The mean value of the scores from the five fields was evaluated (Table 2 and 3 ). 
Table 2: Association between staining intensity and lymph node involvement.

\begin{tabular}{l|ccc}
\hline \multirow{2}{*}{ Staining Intensity } & \multicolumn{2}{|c}{ Lymph node involvement } & p-value \\
\cline { 2 - 3 } & Positive & Negative & \multirow{2}{*}{ 0.0005* $^{*}$} \\
Negative & 7 & 20 & \\
++ Positive & 12 & 3 & 0 \\
+++ Positive & 6 & 0 & 0 \\
\hline
\end{tabular}

Fisher exact test, $p \leq 0.05$, significant

Table 3: Association between staining intensity and lymph node status.

\begin{tabular}{l|ccc}
\hline \multirow{2}{*}{ Staining Intensity } & \multicolumn{2}{c}{ Lymph node status } \\
\cline { 2 - 3 } & $\mathbf{N 1}$ & $\mathbf{N 2}$ & 0 \\
\hline Negative & 7 & 0 & 3 \\
Positive, well differentiated & 4 & 4 & $\mathbf{0 . 0 0 1 *}$ \\
Positive Moderately Differentiated & 0 & 3 & 4 \\
\hline
\end{tabular}

Fisher exact test, $\mathrm{p} \leq 0.05$, significant
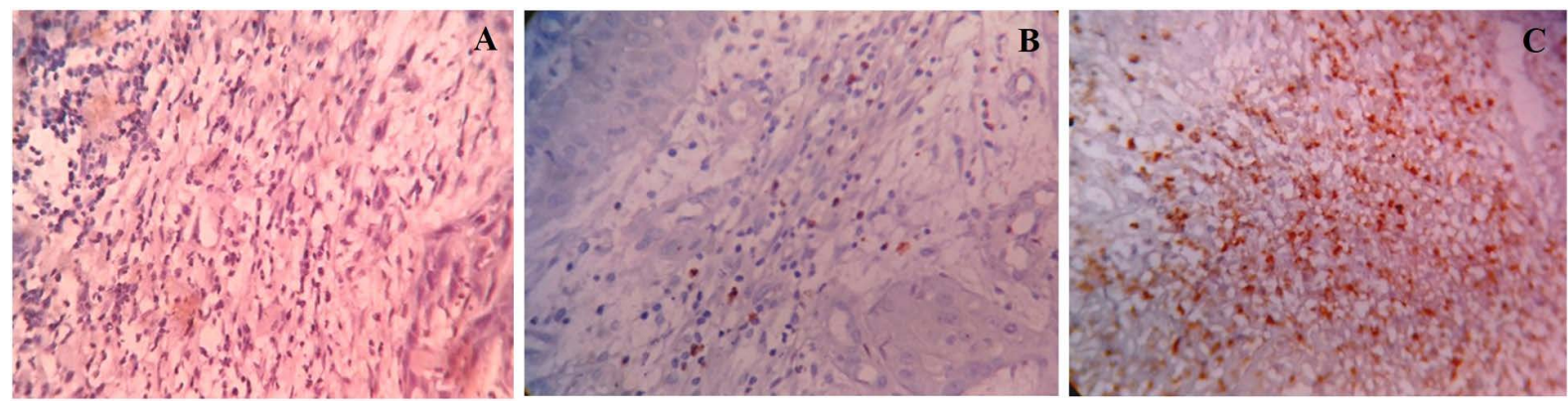

Figure 1: NE immunostaining in OSCC. A- No NE immuno-staining (400 × magnification), B- Mild (1-25\%) NE immuno-staining (400 $\times$ magnification), C- Intense (> 50\%) NE immuno-staining (400 × magnification).

\section{Statistical analysis}

Data collected was analysed using Microsoft Office Excel Package and processed with the SPSS 16.0 software (SPSS Inc., Chicago, USA). Fisher exact test was used to assess the significance of the association between staining intensity and lymph node involvement (positive/negative) and status $(A, B, C)$.

\section{Results}

In the present study, the IHC localization of NE levels were significantly higher $(p$-value $=0.0005)$ in the Group B patients $(61.3 \%)$ with lymph node metastasis as compared to the Group A patients (31.6\%). In the present study, group-A patients with no known lymph node metastasis showed only $5 / 25$ cases positive for Neutrophil Elastase staining. Table 2 and 3 summarizes the results. Habit history revealed that all the patients (with and without lymph node involvement) in the present study had a history of tobacco chewing. There was no statistical difference in the NE expression between stage III and IV.

\section{Discussion}

Neutrophils have an important role in tumour progression, especially with respect to tumour growth and metastasis. ${ }^{[7-9]} \mathrm{NE}$ could effectively decrease TNF signalling resulting in continuous cell growth due to 
the failure of activation of caspase. ${ }^{[8]}$ The role of NE in cancer has largely been attributed to its ability to degrade ECM proteins (collagen, fibronectin, proteoglycan, and cadherins) and especially cause activation of MMP-2, thereby promoting invasion and metastasis. ${ }^{[10,11]}$

The demographic data of the study revealed the predominance of old age and male predilection in the cases reported. The data is in accordance with the earlier studies, which also reported the same predominance regarding age and gender of OSCC cases. ${ }^{[12]}$

A maximum number of cases in the study revealed gingivobuccal complex as the most common site of involvement. The locational predominance is due to the high frequency of tobacco chewing habit in the Indian subcontinent. The predominance of the gingivobuccal complex in OSCC, such cases were referred to as Indian Cancer by Kumar et al. ${ }^{[12]}$ Other common sites of OSCC involvement include the floor of the mouth and ventral surface of the tongue. ${ }^{[14]}$ The cases reported to us for the study were either in stage III or stage IV. The advanced stage of the OSCC in the present study is because most individuals in the Indian subcontinent presenting with the disease report very late to the hospital. But, unlike many studies, most of our cases were moderately differentiated. This could be because half the patients recruited were with lymph node metastasis and were histopathologically aggressive variants. Also, our cases were mostly involving the alveolar and gingivobuccal mucosa, which are known to be more dedifferentiated variants as compared to squamous cell carcinoma of the buccal mucosa. ${ }^{[15]}$ Trellakis et al. ${ }^{[16]}$ detected that CD66 immunostaining correlated with poor patient survival and showed an association between OSCC prognosis and PMN infiltration. These findings were consistent with Wang et al. ${ }^{[17]}$ study wherein the higher clinical stage, recurrence and neutrophil infiltration was associated with lymph node metastasis.

\section{References}

[1] Noy R, Pollard JW. Tumor-associated macrophages: From mechanism to therapy. Immunity 2014;41(1):49-61

[2] Kessenbrock K, Plaks V, Werb Z. Matrix metalloproteinases: regulators of the tumor microenvironment. Cell. 2010;141(1):52-67

[3] Kumar M, Singh PP, Saxena D, et al. Chronic Trauma: As Precipitating Factor of Squamous Cell Carcinoma of Tongue - 3 Case Reports. Indian Journal of Dental Sciences 2014; 4:29-31

[4] Keibel A, Singh V, Sharma MC. Inflammation, microenvironment, and the immune system in
Neutrophil elastase expression predicted a poor clinical outcome that indicated abundant infiltration. The results of the present study are in accordance with Trellakis et al. ${ }^{[16]}$ and Wang et al. ${ }^{[17]}$, which concluded that increased neutrophil infiltration (as elicited by neutrophil elastase marker) was closely associated with local metastasis. Thus, analysing the immuno-expression of NE could aid us in predicting the risk of lymph node metastasis in OSCC. Despite the significant correlation of NE immunostaining with lymph node involvement, there were OSCC cases with lymph node metastasis, which were negative for NE (28\%), and OSCC without lymph node metastasis, which were positive for NE (20\%). Thus, although a positive NE expression increases the risk of a potential lymph node metastasis, it should not be used as a sole criterion.

\section{Conclusions}

The present study indicates a direct correlation between the immuno-expression of NE and lymph node metastasis. Further investigation into the molecular mechanism of NE uptake, antigen processing, and presentation will provide an insight into the role of neutrophil in the initiation and progression of oral cancer.

\section{Conflict of interest}

None

\section{Financial disclosures}

None

cancer progression: These early desmoplastic changes could stimulate fibroblasts and endothelial cell division and produce components for tissue remodelling and neovascularization, ultimately promoting neoplastic processes. Curr Pharm Des 2009; 15: 1949-55.

[5] Bryne M, Koppang HS, Lilleng R, et al. Malignancy grading of the deep invasive margins of oral squamous cell carcinomas has high prognostic value. J Pathol. 1992; 166: 375-81.

[6] Lundqvist L, Stenlund H, Laurell G, et al. The importance of stromal inflammation in squamous 
cell carcinoma of the tongue. J Oral Pathol Med. 2012; 41:379-83.

[7] Kuang DM, Zhao Q, Wu Y, et al. Peritumoral neutrophils link inflammatory response to disease progression by fostering angiogenesis in hepatocellular carcinoma. J. Hepatol. 2011; 54(5):948-55.

[8] Spicer JD, McDonald B, Cools-Lartigue JJ, et al. Neutrophils promote liver metastasis via Mac-1mediated interactions with circulating tumor cells. Cancer Res 2012; 72(16):3919-27

[9] Ho AS, Chen $\mathrm{CH}$, Cheng CC, Wang CC, Lin HC, Luo TY et al. Neutrophil elastase as a diagnostic marker and therapeutic target in colorectal cancers. Oncotarget. 2014;5(2): 473-480

[10] Kim J, Bae J-S. Tumor-Associated Macrophages and Neutrophils in the Tumor Microenvironment. Mediators of Inflammation. 2016:6058147. doi:10.1155/2016/6058147.

[11] Solinas G, Germano G, Mantovani A, et al. Tumorassociated macrophages (TAM) as major players of the cancer-related inflammation. J Leukoc Biol (2009) 86:1065-73

[12] Dissanayaka WL, Pitiyage G, Kumarasiri PV, et al. Clinical and histopathologic: Parameters in the survival of oral squamous cell carcinoma. Oral Surg Oral Med Oral Pathol Oral Radiol 2012;113(4): 518-25.

[13] Kumar M, Nanavati R, Modi T G, et al. Oral Cancer: Etiology and risk factors: a review. J Can Res Ther 2016;12: 458-63

[14] Pires F R, Ramos AB, Oliveira J B C, et al. Oral squamous cell carcinoma: clinicopathological features from 346 cases from a single Oral Pathology service during an 8-year period. J Appl Oral Sci. 2013;21(5):460-7

[15] Sharma M, Madan M, Manjari, et al. Prevalence of Head and Neck Squamous Cell Carcinoma (HNSCC) in our population: The clinic-pathological and morphological description of 198 cases. IJAR 2015; 3(1): 827-833.

[16] Trellakis, S, Farjah, H, Bruderek, et al. Peripheral blood neutrophil: Granulocytes from patients with head and neck squamous cell carcinoma functionally differ from their counterparts in healthy donors. Int $\mathrm{J}$ Immunopathol Pharmacol 2011;24:683-93.

[17] Wang, N, Feng, Y, Wang, Liu S, et al. Neutrophils Infiltration in the Tongue Squamous Cell: Carcinoma and Its Correlation with CEACAM1 Expression on Tumor Cells. PLoS One 2014; 9(2):1-11. 